\title{
Factores que inciden en el destete temprano en un grupo de madres de Santa Marta, Colombia'
}

Gisela González Ruiz²

Luz Ángela Reyes Ríos ${ }^{3}$

Yeis Miguel Borré Ortiz ${ }^{4}$

Haidy Oviedo Córdoba ${ }^{5}$

Loedys Barrios 6

Lorena Carbonó ${ }^{7}$

Gleydis Martínez Quintero8

doi:10.11144/Javeriana.ie17-2.fidt

Cómo citar: González Ruiz G, Reyes Ríos LA, Borré Ortiz YM, Oviedo Córdoba H, Barrios L, Carbonó L, Martínez Quintero G. Factores que inciden en el destete temprano en un grupo de madres de Santa Marta, Colombia. Investig Enferm. Imagen Desarr. 2015;17(2): 37-50. http://dx.doi.org/10.11144/Javeriana.ie17-2.fidt

1. Artículo original de investigación. Recibido: 22 de agosto de 2014. Aceptado: 16 de diciembre de 2014. Disponible en linea: 2 de mayo 2015

2. Enfermera. Magíster en Ciencias Básicas Biomédicas. Profesora Facultad de Enfermería, Universidad Cooperativa de Colombia, sede Santa Marta, Santa Marta, Colombia. Correo electrónico: gisela.1060@gmail.com

3. Enfermera. Magíster en Enfermería Materno Perinatal. Profesora Facultad de Enfermería, Universidad Cooperativa de Colombia, sede Santa Marta. Santa Marta, Colombia. Correo electrónico: luz.reyes@campusucc.edu.co

4. Enfermero. Magíster en Enfermería. Profesor Facultad de Enfermería, Universidad Cooperativa de Colombia, sede Santa Marta, Colombia. yeismiguel@gmail.com

5. Enfermera. Magíster en Enfermeria, Decana Facultad de Enfermeria, Universidad Cooperativa de Colombia, sede Santa Marta. Correo electrónico: haidy.oviedo@ucc.edu.co

6. Enfermeras. Universidad Cooperativa de Colombia, sede Santa Marta. Dittloedys.barrios@, Correo electrónico: loedys.barrios@campusucc.edu.co

7. Enfermera, Universidad Cooperativa de Colombia, sede Santa Marta. Colombia. Correo electrónico: lorena.carbono@campusucc.edu.co

8. Enfermera, Universidad Cooperativa de Colombia, sede Santa Marta, Colombia. Correo electrónico: gleydis.martinez@campusucc.edu.co 


\section{Resumen}

La lactancia materna es la forma ideal de aportar a los niños los nutrientes necesarios para su adecuado crecimiento y desarrollo; sin embargo, muchos factores interrumpen dicha práctica, entre ellos el fenómeno conocido como destete precoz. Objetivo: identificar qué factores inciden para que se presente el destete temprano en un grupo de madres de niños inscritos a un programa de crecimiento y desarrollo de un centro de salud de Santa Marta, Colombia. Metodologia: estudio descriptivo, de corte transversal, cuantitativo en el que se escogió de una población de 372 una proporción de 54 madres con niños menores o iguales a 24 meses, seleccionadas de forma intencional hasta completar la muestra. La información se recolectó utilizando un instrumento diseñado y validado por los investigadores, mediante el juicio de expertos y previa prueba piloto. Resultados: el 94,22\% suministró lactancia materna exclusiva a sus hijos; mientras que el 5,77\% no lo hizo. Los factores que influyeron en la suspensión de la lactancia materna fueron: falta de tiempo (36,54\%), producción insuficiente de leche (23,06\%), rechazo del bebé a la lactancia (17,51\%), decisión propia de la madre $(15,38 \%)$ e influencia familiar (7,69\%). Conclusión: los factores sociales, generalmente, influyen para que se presente el destete temprano; pero la modificación se relaciona con aspectos culturales arraigados como prácticas comunes y costumbres cotidianas. Por ello es necesario que las entidades educativas y de salud continúen realizando esfuerzos que transformen aquello que afecta el bienestar y la salud de diversas poblaciones.

Palabras clave: lactancia materna; destete; recién nacido; nutrición del lactante; madres

\section{Factors affecting early weaning in a group of mothers of Santa Marta, Colombia}

\section{Abstract}

Breastfeeding is the ideal way to give children the nutrients needed for proper growth and development; however, many factors disrupt this practice, including the phenomenon known as early weaning. Objective: To identify what factors contribute to early weaning in a group of mothers of children enrolled in a program of growth and development of a health center in Santa Marta, Colombia. Materials and methods: descriptive, cross-sectional, quantitative study. The sample was comprised by 54 mothers with children less than or equal to 24 months chose from a total of 372 mothers, they were intentionally selected to complete the sample. The information was collected using a questionnaire designed by the researchers, using expert judgment and previous pilot. Results: $94.22 \%$ exclusively breastfed their children; while $5.77 \%$ did not. Factors that influenced the suspension of breastfeeding were lack of time (36.54\%), insufficient milk production (23.06\%), refusal of baby to breastfeeding (17.51\%), Mother's own decision (15.38\%) and family influence (7.69\%). Conclusion: social factors generally influence early weaning; but the change is related to cultural aspects rooted as common practices and everyday habits. It is therefore necessary that educational and health institutions continue their efforts to transform that which affects the welfare and health of diverse populations.

Keywords: breastfeeding; weaning; newborn; infant nutrition; mothers 


\section{Fatores que atingem no desmame precoce numa turma de mães de Santa Marta, na Colômbia}

\section{Resumo}

A aleitamento materno é a forma ideal de fornecer às criancinhas nutrientes necessários para o adequado crescimento e desenvolvimento; no entanto, muito fator interrompe tal prática, incluindo o fenômeno conhecido como desmame precoce. Objetivo: identificar quais os fatores que atingem para que houver desmame precoce numa turma de mães de crianças matriculadas em um programa de crescimento e desenvolvimento de um centro de saúde de Santa Marta, na Colômbia. Materiais e métodos: estudo descritivo, de corte transversal, quantitativo no que foi seleta amostra de 54 mães de uma população de 372 mães com crianças menores ou iguais a 24 meses, escolhidas de forma intencional até completar amostra. A informação foi coletada utilizando um instrumento desenhado e avaliado pelos pesquisadores, mediante parecer de peritos e prévio teste piloto. Resultados: o 94,22\% ministrou amamentação exclusiva aos filhos; enquanto que o $5,77 \%$ não fez assim. Os fatores que influíram na suspensão do aleitamento materno foram: falta de tempo $(36,54 \%)$, produção insuficiente de leite $(23,06 \%)$, o bebê recusou o aleitamento $(17,51 \%)$, decisão própria da mãe $(15,38 \%)$ e influência familiar (7,69\%). Discussão e conclusão: os fatores sociais, geralmente, influenciam para o desmame precoce acontecer; mais a alteração é relacionada com aspectos culturais arraigados como práticas comuns e costumes cotidianas. Por isso é preciso que as entidades educativas e de saúde continuem a realizar esforços para transformar aquilo que afeta o bem-estar e a saúde de diversas populações.

Palavras-chave: aleitamento materno; desmame; recém-nascido; nutrição do lactente; mães 


\section{Introducción}

La lactancia materna exclusiva suministrada el primer año de vida garantiza la salud del recién nacido durante ese periodo y en su vida futura. La Organización Mundial de la Salud (OMS) recomienda lactar de manera exclusiva durante los primeros seis meses de vida, puesto que la lactancia materna es la forma ideal de aportar a los niños los nutrientes necesarios para su adecuado crecimiento y desarrollo $(1,2)$. Esta recomendación ha sido emitida constantemente y ratificada mediante la estrategia mundial para la alimentación del lactante y del niño (3).

La leche materna ayuda a prevenir enfermedades transmitidas por los alimentos, al reducir drásticamente las reglas de preparación y cocción por ser un alimento natural (2). Sin embargo, cuando el lactante ha cumplido los seis meses de vida, se recomienda acudir a la alimentación complementaria (4-6). No obstante, los nutrientes de la leche materna son seguros para el adecuado desarrollo del recién nacido (7). Un estudio comparativo entre leche materna y leche de fórmula evidenció diferencias en el beneficio obtenido por los menores (8), debido a su calidad fisicoquímica y nutritiva (9).

A pesar de que el consumo de leche materna es bandera en programas y estrategias que hacen relevante su importancia, como es el caso de la estrategia de las Instituciones Amigas de la Mujer y la Infancia (IAMI) (10), aún existen dificultades a la hora de lograr una práctica adherente por parte de las madres. La literatura sobre el tema evidencia que la práctica de la lactancia materna exclusiva comúnmente es interrumpida por diversos factores -entre ellos personales, familiares, sociales, culturales e, incluso, biológicos- (11-15), que desencadenan el denominado destete temprano o precoz. Según la Unicef, 17 de cada 100 niños recibía lactancia materna exclusiva en 1990; pero este valor descendió a 11,6 en el $2000(16,17)$. No obstante, en Colombia, entre el 2005 y el 2010 se detuvo el crecimiento de la lactancia materna, la exclusiva descendió del $47 \%$ al $45 \%$ y la duración total se mantuvo en 15 meses (18), lo cual evidencia un aumento significativo en las cifras de abandono de la lactancia materna.

En estudios previos se ha identificado un considerable porcentaje de madres que lactan menos de cinco meses (19), debido al desconocimiento sobre lactancia materna y las técnicas de amamantamiento (20). Algunos autores plantean que este fenómeno se relaciona directamente con el grado o nivel de escolaridad y la vinculación laboral de la mujer (21-26).

En varios estudios observacionales y comparativos se ha encontrado que los niños alimentados con lactancia materna exclusiva durante los seis primeros meses de vida tienen menos probabilidad de enfermar frente aquellos niños que han sido amamantados durante menos de seis meses (27-29).

El destete temprano es un fenómeno que varía de región en región, con un alto componente biocultural $(30,31)$. La literatura muestra que las razones comúnmente referidas por las madres para abandonar la lactancia materna exclusiva son la baja producción de leche, problemas de agarre y dolor $(20,32)$.

En Colombia, según cifras del Ministerio de Salud y Protección Social, el promedio de duración total de lactancia materna para el 2010 fue de 14,9 meses, y la lactancia materna exclusiva fue de 1,8 meses (33). 
Según recomendaciones de la Organización Panamericana de la Salud y el Ministerio de Salud y Protección Social de Colombia, es importante que se investiguen y exploren las prácticas que conllevan al destete temprano en Colombia (18).

Camargo y colaboradores (34) recomiendan fortalecer la intervención del personal de salud en las etapas relacionadas con la lactancia materna, en los diferentes programas de atención primaria, entre ellos los programas de crecimiento y desarrollo. En Santa Marta no existen evidencias de estudios similares en los que se haya abordado el tema del abandono de la lactancia materna. Por tanto, la identificación y el conocimiento de los factores que inciden para que este problema se presente permite diseñar y establecer estrategias contextualizadas de intervención que conduzcan a orientar y ayudar a las madres a reconocer la importancia de este proceso para la vida de su bebé.

El presente estudio tuvo como objetivo identificar los factores que inciden para que se presente el destete temprano en un grupo de madres de niños inscritos en un programa de crecimiento y desarrollo, de un centro de salud de Santa Marta, Colombia.

\section{Metodología}

Estudio descriptivo, de corte transversal, con abordaje cuantitativo. La muestra estuvo conformada por madres asistentes a los controles de crecimiento y desarrollo de sus hijos, durante el segundo periodo del 2013.

De una población de 372 madres, se escogió una proporción de 54 con niños menores o iguales a 24 meses que no estaban lactando en el momento del estudio y que decidieron participar en la investigación de forma voluntaria, firmando el consentimiento informado. Se excluyeron 6 madres con periodos intermitentes de lactancia materna, y 10, que no dieron su consentimiento para participar en el estudio.

Las participantes se seleccionaron mediante muestreo intencional, no probabilístico, hasta completar la muestra de madres con historial de abandono de lactancia materna.

La recolección de los datos se llevó a cabo en dos fases: la primera, mediante los registros clínicos de los hijos, existentes en el programa, y la segunda, a través de la aplicación de una encuesta directamente a las madres. Dicha encuesta fue diseñada por el equipo de investigación con base en la revisión de literatura; posteriormente, fue enviada a cinco profesionales con formación de especialización y maestría en el área materno-infantil para su revisión y sugerencias, y finalmente, evaluada mediante prueba piloto en población con similares características. La estructura interna de la encuesta estaba conformada por variables de interés que se organizaron en dos apartados: a) información sociodemográfica y b) causas, motivos y razones de abandono de la lactancia materna.

Durante la investigación se preservaron aspectos éticos emitidos en la Resolución 8430 de 1993 (35) y la Declaración de Helsinki (36). Además, se utilizó el consentimiento informado como medida de respeto a la autonomía de las participantes y se tuvo el aval del comité de ética de la Universidad 
Cooperativa de Colombia. Finalmente, la información recolectada fue organizada, tabulada y procesada en el programa de Microsoft Excel®, analizada con estadística descriptiva y graficada mediante histograma y diagrama de sectores.

\section{Resultados}

De las 54 madres participantes del estudio, el 40,7\% está entre 15 y 25 años de edad; $52 \%$, entre 26 y 35 años, y el 1,8\% es mayor de 35 años. En cuanto al estado civil, se observó que $22,2 \%$ son casadas, $40,7 \%$ son solteras y $33,3 \%$ convive en unión marital libre.

El 64,8\% vive en estrato socioeconómico 1; el 27,7\%, en estrato 2, y el 3,37\%, en estrato 3 . El $61,1 \%$ se dedica al cuidado del hogar, el 11,1\% labora de manera dependiente, el 7,4\% estudia y el 16,6\% estudia y trabaja simultáneamente.

Con respecto al nivel de escolaridad, se halló que el 42,59\% cursó o está cursando estudios de básica primaria; el 48,98\%, estudios secundarios o técnicos, y solo el 8,4\% logró estudios completos o incompletos de nivel universitario.

La edad de los recién nacidos osciló entre los 2 y los 24 meses. El 59,6\% es de sexo femenino y el $40,38 \%$ es de sexo masculino. Al nacer, el 51,92\% tuvo un peso que osciló entre 2500 y 4000 gramos; el 30,77\%, un peso inferior a 2500 gramos, y el 17,31\% un peso superior a 4000 gramos (figura 1).

Del total de las madres encuestadas, se observó que de forma general el 94,22\% ha suministrado lactancia materna a sus hijos en algún momento; mientras que el 5,77\% no lo ha hecho. El tiempo de suministro de la lactancia se distribuyó de la siguiente forma: el 50\% lo hizo durante 3 a 6 meses; el 21,1\%, de 7 a 12 meses, el 13,4\%, más de un año, y el 9,6\%, menos de 3 meses.

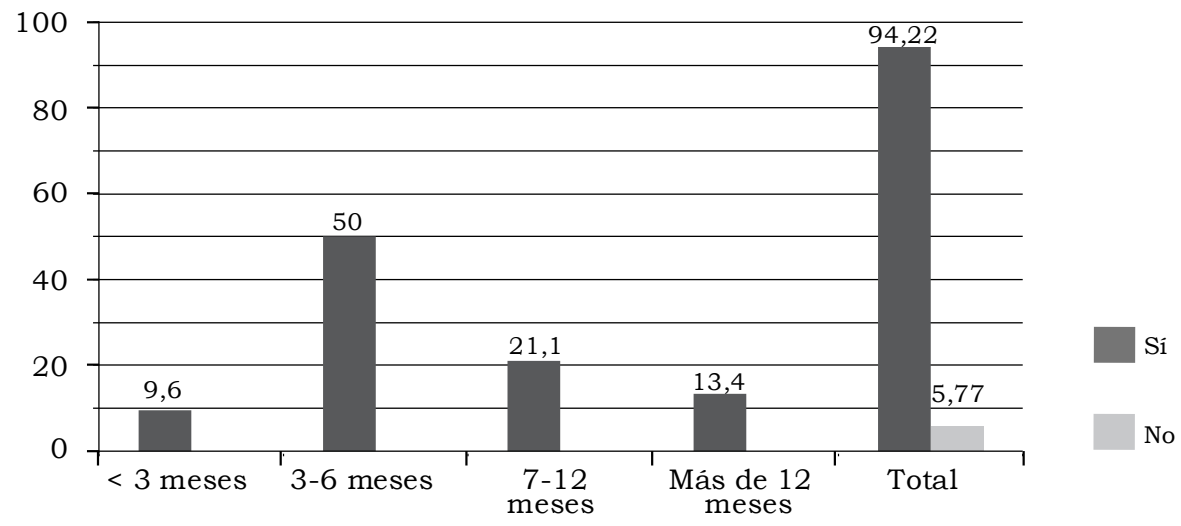

Figura 1. Tiempo de suministro de lactancia materna exclusiva y complementaria

Fuente: Encuesta aplicada a las madres, 2013 
La figura 2 muestra que los factores que, según las madres, influyeron en el abandono de la lactancia materna fueron: falta de tiempo para lactar, producción insuficiente de leche, rechazo del bebé a la lactancia, decisión de la madre y orientaciones dadas por terceros (familiares y amigos). La falta de tiempo para lactar y la decisión propia de las madres en destetar a su hijo podria estar asociada con la ocupación, la imagen o la actividad laboral desempeñada. Sin embargo, esta correlación podría hacerse en futuros estudios que se lleven a cabo en la región.

La figura 3 muestra que la incorporación de alimentación complementaria es un aspecto de alta incidencia en la práctica de las madres encuestadas. El 84,6\% de las participantes lo hizo durante los 3 primeros meses de vida del recién nacido. Los alimentos principalmente proporcionados fueron: leche de fórmula, en un 26\%; purés/papillas y sopas, en un 25\%; mazamorra, coladas y jugo de frutas, en un $23 \%$. La distribución porcentual del tipo de leche suministrado después del destete correspondió a leche de fórmula, en un $48 \%$; leche de vaca, en un $42,3 \%$, y leche de soya, en un $9,6 \%$.

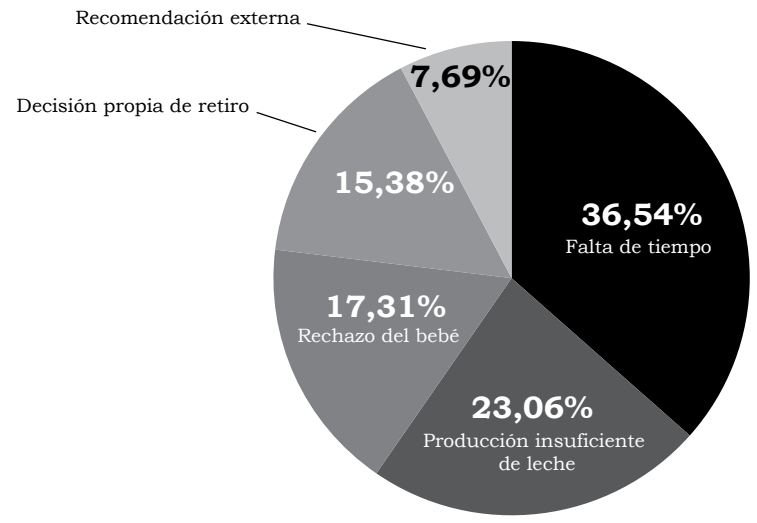

Figura 2. Factores presentes en el abandono de la lactancia materna

Fuente: Encuesta aplicada a las madres, 2013

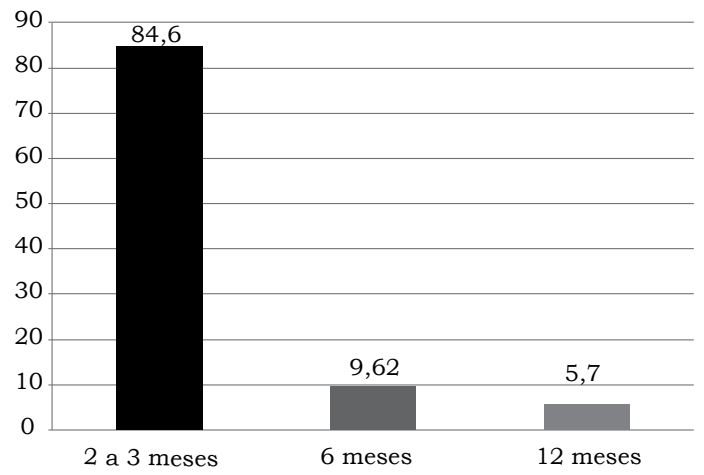

Figura 3. Tiempo de incorporación de alimentación complementaria

Fuente: Encuesta aplicada a las madres, 2013 


\section{Discusión}

Un aspecto importante observado en este estudio tiene que ver con los embarazos adolescentes. Se encontró que el 40,7\% de las madres participantes tenía edades comprendidas entre los 15 y 25 años, lo cual es coherente con lo hallado en estudios previos $(20,37,38)$. Por ejemplo, Borré y cols. (20) hallaron que el $51 \%$ de las madres estudiadas tenía entre 15 y 25 años de edad. Pinilla y colaboradores (37) encontraron que la edad promedio de las madres adolescentes fue de 17,9 años. Veramendi y colaboradores (38) observaron que el $32,4 \%$ de la población estudiada correspondió a embarazadas adolescentes.

El anterior aspecto es de suma importancia, porque este hallazgo corrobora las estadísticas nacionales e internacionales, en las cuales se indica que la incidencia de embarazos en adolescentes se ha convertido en un importante problema de salud pública mundial (37). De hecho, según Estrada y cols. (39), el embarazo adolescente es uno de los factores que se relaciona de manera directa con el destete precoz o abandono de la lactancia materna.

Con respecto a lo anterior, es importante resaltar que, a pesar de que el Ministerio de Educación colombiano ha intentado incluir formación sobre educación sexual y reproductiva en la educación primaria y secundaria, los resultados han sido poco evidentes, debido a que el problema de embarazo en adolescentes sigue creciendo de manera descomunal. Además, afecta todos los ámbitos de la adolescente, incluido el escolar. Es conocido que una adolescente embarazada, muchas veces, se convierte en una desertora de la escuela; pero, de igual forma, una adolescente con baja escolaridad tiene mayor probabilidad de quedar embarazada a temprana edad.

De hecho, son varias las investigaciones que han demostrado la incidencia de la escolaridad en el conocimiento que puedan tener o no tener las madres sobre la importancia de la lactancia materna para sus hijos $(13,14,37,40)$. Tal realidad guarda coherencia con lo hallado en el presente estudio, en el cual, aunque no se realizaron pruebas de correlación entre estas variables, presuntivamente se hace visible por el factor mismo de destete y el grado de conocimientos de la madres. Esta limitante puede ser mejorada mediante la realización de nuevos estudios en los que se correlacionen dichas variables a través de técnicas estadísticas de rigor.

Por otra parte, el porcentaje de madres que lactaron durante un periodo menor a 6 meses es un factor de riesgo que ha estado presente en otros estudios, como causa asociada a enfermedades futuras en los niños $(19,29)$.

De acuerdo con los resultados del presente estudio, los factores presentes en el destete temprano en la población estudiada guardan relación con el estudio de Niño y cols. (41) y López y cols. (42), quienes encontraron factores similares que causaban el abandono de la lactancia materna, tanto en el sector de salud público como en el privado. Entre ellos enfermedades del niño y de la madre, rechazo del pecho, decisión propia de las madres, inicio de vida escolar o laboral, producción insuficiente de leche y cuestiones estéticas. 
Sin embargo, existen estudios con resultados diferentes al presente, en los cuales se observa que los factores relacionados con un mayor abandono de la lactancia materna son: deficiencia de conocimientos sobre lactancia materna; información brindada por el personal de salud; estrato socioeconómico bajo; uso de chupetes, pezoneras o biberón $(34,43,44)$; malas experiencias pasadas (34,45); nivel de estudios (21-26,43,44); gestación tras técnicas de reproducción asistida; hábito tabáquico materno; expectativas pobres sobre la duración de la lactancia (44); tipo de parto; falta de apoyo de la pareja (45), y sensación de que el niño queda con hambre $(45,46)$.

Al confrontar estos hallazgos con las actividades realizadas por las madres encuestadas, se encontró que el $61,1 \%$ se encarga de las actividades cotidianas en el hogar, situación que supone la existencia de mayores oportunidades para adherirse al proceso de lactancia y un adecuado manejo del tiempo. En este sentido, la Unicef expresa que todas las madres pueden amamantar si se les brinda el apoyo, la consejería, la motivación adecuada y la ayuda práctica para la resolución de problemas (47); proceso en el cual los profesionales de la salud cumplen un rol importante, especialmente enfermería (48).

Este estudio corrobora que el destete temprano o precoz se produce por dos aspectos: por factores del niño y por factores maternos. Sin embargo, es más frecuente la presencia de factores maternos que del niño. En los niños, el factor más común es el rechazo a la lactancia materna. Vásquez (49) afirma que este rechazo es un factor que puede darse por enfermedad, fastidio, comprobación de diferencia entre el biberón, el pezón, entre otros.

Al analizar los resultados del presente estudio, se observan los porcentajes de casos que están por fuera de las recomendaciones internacionales y nacionales respecto a la lactancia materna, situación que coincide con lo expresado por la OMS, la Unicef y el Ministerio de Salud y Protección Social en las estadísticas mundiales y de Colombia, respectivamente (33).

Por otra parte, los altos porcentajes en cuanto a la alimentación complementaria a edades tempranas observados en este estudio ponen de manifiesto el riesgo de enfermedad que podría sufrir esta población infantil en próximos años, tal como se ha demostrado en estudios previos $(19,29)$. No obstante, cabe resaltar que la ingesta de alimentación complementaria no es que sea inadecuada, pero debe darse en el tiempo estipulado y de acuerdo con la madurez del sistema biológico del recién nacido.

A través de los años, en las regiones de la costa caribe colombiana se ha arraigado la creencia que apoya la inserción de alimentos a temprana edad. Tal creencia se ha trasmitido de generación en generación y los familiares la han aceptado como buenas prácticas. Según Leininger, la cultura es el conocimiento que se adquiere y trasmite, con sus valores, creencias, reglas de comportamiento y prácticas en estilo de vida, que orientan estructuralmente a un grupo determinado en sus pensamientos y actividades (50).

Dichas prácticas se fundamentan en mitos relacionados con el crecimiento y desarrollo del niño. Es decir, a mayor cantidad de alimentos suministrados al niño, mayores posibilidades de tener hijos que crezcan sanos y 
fuertes. Dicha situación resalta no solo el poder de las creencias, hábitos y factores culturales sobre los resultados nutricionales de la población, sino que también evidencia las necesidades educacionales que requiere la población para el mejoramiento de los estilos y hábitos de vida. Este fuerte componente cultural ha sido denominado por Jiménez y Valencia (30) y Paricio (51) como fenómeno biocultural.

\section{Conclusión}

Esta investigación muestra un panorama contextualizado de los diferentes factores que influyen para que se presente la práctica del destete temprano en la población estudiada. Sin embargo, la modificación de dichos factores es una cuestión que no solo compromete aspectos de conocimiento y educación, sino que también relaciona aspectos culturales que se han arraigado como prácticas comunes y costumbres cotidianas propias de sus hábitos de vida. Por tanto, se convierte en una evidencia que servirá de referencia para futuros estudios de investigación e intervenciones familiares y comunitarias dirigidas a esta población.

\section{Limitaciones del estudio}

Los próximos estudios sobre el tema deben superar las limitaciones del diseño del presente trabajo. Por ejemplo, la selección de los participantes, las pruebas de correlación entre variables y la validación de contenido del instrumento para la recolección de la información.

\section{Recomendaciones}

Es necesario que las entidades educativas y de salud sigan realizando esfuerzos que permitan la transformación cultural de aquello que afecta el bienestar y la salud de diversas poblaciones, de manera que los abordajes culturales sean congruentes con el deber ser que evoca la práctica científica.

Para apoyar este propósito, sería conveniente que las empresas promotoras de salud y las secretarias de salud, junto con las secretarías de educación distritales y municipales, incluyeran información y educación sobre estos temas en los programas de formación académica.

Por otra parte, es importante que la publicidad implacable de los laboratorios a través de los medios de comunicación tenga un indispensable componente de racionalización sobre la importancia de la lactancia materna natural, puesto que sería contraproducente incentivar la adherencia a la práctica de la lactancia materna cuando los medios de comunicación les dan un alto valor a las prácticas artificiales.

Por último, es necesario que se continúe con la validación del instrumento, mediante pruebas de contenido, de criterio, de constructo y de confiabilidad.

\section{Conflicto de intereses}

Los autores declaran no tener conflicto de intereses. 


\section{Financiación}

Ninguna

\section{Referencias}

1. Organización Mundial de la Salud. Lactancia materna [internet]. [Citado 30 may 2014]. [aprox. 2 pantallas]. Disponible en: http:// www.who.int/maternal_child_adolescent/topics/newborn/nutrition/ breastfeeding/es/

2. Organización Mundial de la Salud, Departamento de Inocuidad de los Alimentos, Zoonosis y Enfermedades de Transmisión Alimentaria. Manual sobre las cinco claves para la inocuidad de los alimentos. Francia: OMS; 2007.

3. Organización Mundial de la Salud. Estrategia mundial para la alimentación del lactante y del niño pequeño. Ginebra: OMS; 2003.

4. Araya $Z$, Brenes G. Educación prenatal: factor asociado al exito de lactancia materna. Rev Enfermería Actual en Costa Rica [internet]. 2013 [citado 21 Jun 2014];23: [aprox. 15 pantallas]. Disponible en: http://www.revenf.ucr.ac.cr/lactanciamaterna.pdf

5. Asociación Nacional de Pediatría de España. Recomendaciones sobre lactancia materna del comité de lactancia materna de la Asociación Española de Pediatría [internet]. [Citado 21 jun 2014]. [aprox. 17 pantallas]. Disponible en: http://www.aeped.es/sites/default/files/recomendaciones_lm_26-1-2012.pdf

6. Latham MC. Lactancia materna. En: Latham MC. Nutrición humana en el mundo en desarrollo. New York: Colección FAO; 2002.

7. Díaz-Argüelles V. Lactancia materna: evaluación nutricional en el recién nacido. Rev Cubana Pediatr [internet]. 2005 Jun [citado 2014 Nov 23];77(2). Disponible en: http://scielo.sld.cu/scielo. php?script=sci_arttext\&pid=S0034-75312005000200005\&lng $=$ es

8. Sevilla R, Zalles L, Santa Cruz W. Lactancia materna vs nuevas fórmulas lácteas artificiales: Evaluación del impacto en el desarrollo, inmunidad, composición corporal en el par madre/niño. Gac Med Bol. 2011;34(1):6-10.

9. López M, Blanes M, Herrera M, Mora C. Estudio de la calidad fisicoquímica y microbiológica de la leche humana colectada por el banco de leche del Hospital Infantil San Pablo. Pediatr (Asunción). 2013;40(3):253-60. Disponible en: http://scielo.iics.una.py/scielo. php?script $=$ sci_arttext\&pid=S1683-98032013000300008\&lng=es\& $\mathrm{nrm}=$ iso

10. Ministerio de la Protección Social. Criterios globales para el cumplimiento de los 10 pasos de la iniciativa instituciones amigas de la mujer y la infancia, IAMI integral. Bogotá: MPS-Unicef; 2008.

11. Belfor RR, Ocaña V. Factores biopsicosociales que influyen en la lactancia materna de neonatos atendidos en hospitalización del área gineco-obstetricia del Hospital León Becerra, Milagro [tesis]. Milagro (Ecuador). Universidad Estatal de Milagro; 2011. Disponible en: 
http://repositorio.unemi.edu.ec/bitstream/123456789/369/1/5\%20 FACTORES \%20BIOPSICOSOCIALES.pdf

12. Londoño A, Mejía M. Factores relacionados con lactancia materna exclusiva e introducción temprana de leche entera en comunidades de estrato socioeconómico bajo. Calarcá-Colombia. Rev Gerenc Polit Salud. 2010;9(19):124-37.

13. Alonso R, Fariñas A, Rodríguez B, Campo A, Díaz R. Intervención educativa sobre la atención a niños de madres adolescentes. Hospital Ginecoobstétrico Guanabacoa. 2002-2008. Index Enferm. 2010;19(4):289-93.

14. Llamuca MM. Factores que inciden en el abandono de la lactancia materna en los primeros 6 meses de las madres adolescente, en el subcentro de salud "Los Vergeles" de la ciudad de Milagro [tesis]. Milagro (Ecuador). Universidad Estatal de Milagro; 2013. Disponible en: http://repositorio.unemi.edu.ec/bitstream/123456789/1521/1/ tesis \%20final.pdf

15. Avalos MM. Comportamiento de algunos factores biosociales en la lactancia materna en los menores de un año. Rev Cubana Med Gen Integr. 2011;27(3):323-31.

16. Fondo de las Naciones Unidas para la Infancia (Unicef). Por una niñez bien nutrida: comunicación para la acción. 3ra ed. Bogotá: Unicef; 2004.

17. Organización Mundial de la Salud (OMS). Temas de nutrición: la alimentación complementaria [internet]. [Citado 20 Jun 2014]. [aprox. 3 pantallas]. Disponible en: http://www.who.int/nutrition/topics/complementary_feeding/es/index.html

18. Ochoa G. Avances y retos de la lactancia materna en Colombia 1970 a 2013: un recorrido sobre 40 años de experiencias en la aplicación de politicas públicas. Washington, D. C.: OPS; 2013.

19. Flores-Díaz AL, Bustos-Valdés MV, González-Solís R, Mendoza-Sánchez HF. Factores relacionados con la duración de la lactancia materna en un grupo de niños mexicanos. Arch Med Famil. 2006;8(1):33-9.

20. Borré YM, Cortina C, González G. Lactancia materna exclusiva: ¿la conocen las madres realmente? Rev Cuid. 2014;5(2):723-30.

21. Briere CE, McGrath J, Cong X, Cusson R. An integrative review of factors that influence breastfeeding duration for premature infants after NICU hospitalization. J Obst Gynecol Neo Nurs. 2014;43(3):272-81.

22. Cadena JM, Enríquez SV. Factores relacionados al destete precoz y los conocimientos, creencias y actitudes respecto de la lactancia exclusiva en madres con niños menores a 6 meses de vida que acuden al centro de salud $\mathrm{N}^{\circ} 1$ de la ciudad de Tulcán durante el periodo marzo-agosto del 2012 [tesis]. Tulcán (Ecuador). Universidad Politécnica Estatal del Carchi en convenio con Universidad Central del Ecuador; 2012. Disponible en: http://181.198.77.140:8080/handle/123456789/211

23. Mastrup R, Hansen BM, Kronborg H, Norby S, Hallum K, Frandsen A, et al. Factors Associated with Exclusive Breastfeeding of Preterm 
Infants. Results from a Prospective National Cohort Study. PLoS ONE. 2014;9(2):e89077.

24. Rius JM, Ortuño J, Rivas C, Maravall M, Calzado MA, López A, et al. Factors associated with early weaning in a Spanish region. Anales de Pediatría. 2014;80(1):6-15.

25. Cardona K, Castaño JJ, Hurtado CI, Moreno MI, Restrepo A, Villegas OA. Frecuencia de lactancia materna exclusiva, factores asociados y relación con el desarrollo antropométrico y patologías en una población de lactantes atendida en ASSBASALUD ESE, Manizales (Colombia) 2010-2011. Arch Med (Manizales). 2013;13(1):73-87.

26. Espinoza C, Zamorano CA, Graham S, Orozco A. Factores que determinan la duración de la lactancia materna durante los tres primeros meses de vida. An Med (Mex). 2014;59(2):120-6.

27. De la Vega T, Pérez VT, Bezos L. La lactancia materna y su influencia en el comportamiento de las infecciones respiratorias agudas. Rev Cubana Med Gen Integr. 2010;26(3):483-9.

28. Moraga F. Lactancia materna y postnatal, un desafío de país. Rev Chil Pediatr. 2011;82(4):273-5.

29. Fernández EJ, Lemes GS, Alum JM, Díaz Y. Estado de salud de los niños de 8 a 14 meses según el tipo de lactancia materna. Rev Cubana Med Gen Integr. 2014;30(1):82-92.

30. Jiménez R, Valencia M. Epidemiología de la lactancia materna. Rev Méd La Paz. 2009;15(1):71-4.

31. Odom EC, Li R, Scanlon KS, Perrine CG, Grummer-Strawn L. Reasons for earlier than desired cessation of breastfeeding. Pediatrics. 2013;131(3):e726-32.

32. Stuebe AM. Formas de facilitar que la mujer alcance sus metas de lactancia materna. Obstet Gynecol. 2014;123:643-52.

33. Ministerio de Salud y Protección Social. Colombia necesita mejorar la lactancia materna. Boletín de Prensa 288 [internet]. 2013 Ago [citado 2014 Sep 30]. Disponible en: http://www.minsalud.gov.co/Paginas/ lactancia-materna.aspx

34. Camargo FA, Latorre JF, Porras JA. Factores asociados al abandono de la lactancia materna exclusiva. Hacia la Promoción de la Salud. 2011;16(1):56-72.

35. Resolución 008430 de 1993, por la cual se establecen las normas científicas, técnicas y administrativas para la investigación en salud. Ministerio de Salud de Colombia; 1993.

36. Mazzanti MA. Declaración de Helsinki, principios y valores bioéticos en juego en la investigación médica con seres humanos. Rev Colomb Bioética. 2011;6(1):125-44.

37. Pinilla E, Domínguez CC, García A. Madres adolescentes, un reto frente a los factores que influyen en la lactancia materna exclusiva. Rev Enferm Glob. 2014;13(1):59-70.

38. Veramendi-Espinoza LE, Zafra-Tanaka JH, Ugaz-Soto LM, VillaSantiago NM, Torres-Bravo LA, Pinto-Arica AA, et al. Conocimientos, 
actitudes y prácticas de lactancia materna en madres de niños de comunidad rural peruana y su asociación con la diarrea aguda infecciosa. CIMEL. 2012;17(2):82-8.

39. Estrada J, Amargós J, Reyes B, Guevara A. Intervención educativa sobre lactancia materna. Rev AMC. 2010;14(2).

40. Grau PMD. Morbilidad en el lactante de seis meses y su relación con la alimentación. Rev Cub Pediatr. 2005;77(1):1.

41. Niño R, Silva G, Atalah E. Factores asociados a la lactancia materna exclusiva. Rev Chil Pediatr. 2012;83(2):161-9.

42. López BE, Martínez LJ, Zapata NJ. Motivos del abandono temprano de la lactancia materna exclusiva: un problema de salud pública no resuelto en la ciudad de Medellín. Rev Fac Nac Salud Pública. 2013;31(1):117-26.

43. Oliver Roig A, Richart Martínez M, Cabrero García J, Pérez Hoyos S, Laguna Navidad G, Flores Álvarez JC, et al. Factores asociados al abandono de la lactancia materna durante los primeros 6 meses de vida. Rev Latino-Am Enfermagem [internet]. 2010 [citado 10 Oct 2014];18(3): [09 pantallas]. Disponible en: http://www.scielo.br/pdf/rlae/v18n3/es_12.pdf

44. Rius JM, Ortuño J, Rivas C, Maravall M, Calzado MA, López A, et al. Factores asociados al abandono precoz de la lactancia materna en una región del este de España. An Pediatr (Barc). 2013;8(1):6-15.

45. Félix JA, Martínez E, Sotelo EI. Prevalencia y factores biosociales asociados al abandono de la lactancia materna exclusiva. Pediatría de México. 2011;13(2):47-56.

46. Navarro-Gil C. Prevalencia de lactancia materna y causas de abandono en los dos primeros meses de vida, en las Comarcas de La Jacetania y Alto Gállego. Med Naturista. 2013;7(2):61-5.

47. Fondo de las Naciones Unidas para la Infancia (Unicef). Nutrición: lactancia materna [internet]. [Citado 19 Sep 2014]. [aprox. 4 pantallas]. Disponible en: http://www.unicef.org/spanish/nutrition/index_24824.html

48. Dos Santos MM, Andrade F, Ávila IE, Magalhaes EM. El rol del profesional de enfermería frente a la lactancia materna en la madre con VIH. Rev Cuid. 2012;3(3):404-9.

49. Vásquez M. El bebé no quiere la leche materna, ¿qué hacer? [Internet]. 2013 [citado 2014 May 25]. Disponible en: http://www.consumer.es/ web/es/bebe/lactancia/2013/01/30/215569.php

50. Leininger M. Culture care diversity and universality theory and evolution of the ethnonursing method. En: Leininger M, Mcfarland M. Culture care diversity and universality. $2^{\text {nd }}$ ed. Massachusetts: Jones And Bartlett Publishers; 2006. p. 1-43.

51. Paricio J. Aspectos históricos de la alimentación al seno materno. En: Comité Lactancia Materna AEP Lactancia Materna. Guía para profesionales. Barcelona; 2004. p. 7-25. 